\title{
Interstitial Oxide Ion Distribution and Transport Mechanism in Alu- minum-doped Neodymium Silicate Apatite Electrolytes
}

Tao An, ${ }^{\dagger, 1}$ Tom Baikie, ${ }^{\ddagger}$ Alodia Orera, ${ }^{\sigma}$ Ross O. Piltz, ${ }^{\S}$ Martin Meven, ${ }^{\perp}$ Peter R. Slater, ${ }^{\boldsymbol{\top}}$ Jun Wei, ${ }^{*, \#}$ María L. Sanjuán, ${ }^{\sigma}$ and T. J. White ${ }^{* \dagger}$

${ }^{\dagger}$ Nanyang Technological University, School of Materials Science and Engineering, 50 Nanyang Avenue, Singapore 639798, Singapore, ${ }^{\ddagger}$ Energy Research Institute @ NTU (ERI@N), Nanyang Technological University, Singapore 637553, Singapore, ${ }^{\circ}$ ICMA, CSIC-Universidad de Zaragoza, 50009 Zaragoza, Spain, ${ }^{\S}$ Bragg Institute, Australian Science and Technology Organisation, Lucas Heights, NSW 2234, Australia, ${ }^{\perp}$ RWTH Aachen, Institute of Crystallography and Jülich Centre for Neutron Sciences(JCNS) at Heinz Maier-Leibnitz Zentrum (MLZ), Lichtenbergstraße 1, 85747 Garching, Germany, 'School of Chemistry, University of Birmingham, Edgbaston, Birmingham, B15 2TT, UK, and "Singapore Institute of Manufacturing Technology (SIMTech), Agency for Science, Technology and Research (A*STAR), 71 Nanyang Drive, 638075, Singapore. ${ }^{1}$ Present address: Institute of Materials Research and Engineering (IMRE), Agency for Science, Technology and Research (A*STAR), 2 Fusionopolis Way, Innovis, \#o8-03, Singapore 138634, Singapore. * Email: tjwhite@ntu.edu.sg; jwei@simtech.a-star.edu.sg.

\section{RAMAN ACTIVE MODES}

For $\mathrm{Nd}_{9 \cdot 33} \mathrm{Si}_{6} \mathrm{O}_{26}$, the total number of expected Raman active modes is $63\left(21 \mathrm{~A}+21 \mathrm{E}_{1}+21 \mathrm{E} 2\right)$ in $P 6_{3}$ and $33\left(12 \mathrm{~A}_{\mathrm{g}}+8 \mathrm{E}_{1 \mathrm{~g}}+13 \mathrm{E}_{2 \mathrm{~g}}\right)$ in $P 6_{3} / m$ space groups, respectively, while each split oxygen site leads to extra $9\left(3 \mathrm{~A}+3 \mathrm{E}_{1}+3 \mathrm{E}_{2}\right)$ modes in $P 6_{3}$ and $9\left(3 \mathrm{~A}_{g}+3 \mathrm{E}_{1 \mathrm{~g}}\right.$ $\left.+3 \mathrm{E}_{2 \mathrm{~g}}\right)$ modes in $P 6_{3} / \mathrm{m}$. Therefore, the total expected Raman active modes is $90\left(30 \mathrm{~A}+30 \mathrm{E}_{1}+30 \mathrm{E}_{2}\right)$ in $P 6_{3}$ and ${ }_{51}\left(18 \mathrm{Ag}_{\mathrm{g}}+14 \mathrm{E}_{1 \mathrm{~g}}\right.$ $+19 \mathrm{E}_{2 \mathrm{~g}}$ ) in $P 6_{3} / \mathrm{m}$ space groups, respectively, in accordance with the split of oxygen sites $\mathrm{O}(1)^{\prime}, \mathrm{O}(3)^{\prime}$ and $\mathrm{O}(4)^{\prime}$ 'in $P 6_{3}$ (Table 1 ) and $\left.\mathrm{O}_{1}\right)^{\prime}$ and $\left.\mathrm{O}_{3}\right)^{\prime}$ in $\mathrm{P6}_{3} / \mathrm{m}$ (Table 2 ) as determined by the neutron diffraction performed at $2 \mathrm{~K}$. For internal modes of unperturbed $\mathrm{SiO}_{4}, 27$ modes are expected for $P 6_{3}$ and 15 are expected for $P 6_{3} / \mathrm{m}$.

Table S1. Raman activity in $\mathrm{Pb}_{3}$ space group.

\begin{tabular}{lccc}
\hline Atom & Site symmetry & Raman activity & Number of modes \\
\hline $\mathrm{Nd}(1)$ & $2 b$ & $\mathrm{~A}+\mathrm{E}_{1}+\mathrm{E}_{2}$ & 3 \\
$\mathrm{Nd}(2)$ & $2 b$ & $\mathrm{~A}+\mathrm{E}_{1}+\mathrm{E}_{2}$ & 3 \\
$\mathrm{Nd}(3)$ & $6 c$ & $3 \mathrm{~A}+3 \mathrm{E}_{1}+3 \mathrm{E}_{2}$ & 9 \\
$\mathrm{Si}$ & $6 c$ & $3 \mathrm{~A}+3 \mathrm{E}_{1}+3 \mathrm{E}_{2}$ & 9 \\
$\mathrm{O}(1)$ & $6 c$ & $3 \mathrm{~A}+3 \mathrm{E}_{1}+3 \mathrm{E}_{2}$ & 9 \\
$\mathrm{O}(2)$ & $6 c$ & $3 \mathrm{~A}+3 \mathrm{E}_{1}+3 \mathrm{E}_{2}$ & 9 \\
$\mathrm{O}(3)$ & $6 c$ & $3 \mathrm{~A}+3 \mathrm{E}_{1}+3 \mathrm{E}_{2}$ & 9 \\
$\mathrm{O}(4)$ & $3 \mathrm{~A}+3 \mathrm{E}_{1}+3 \mathrm{E}_{2}$ & 9 \\
$\mathrm{O}(5)$ & $6 c$ & $\mathrm{~A}+\mathrm{E}_{1}+\mathrm{E}_{2}$ & 3 \\
\hline & $2 a$ & Total (without split sites): & 63 \\
\hline $\mathrm{Split}$ site & & Raman activity & Number of modes \\
\hline $\mathrm{O}(1)^{\prime}$ & Site symmetry & $3 \mathrm{~A}+3 \mathrm{E}_{1}+3 \mathrm{E}_{2}$ & 9 \\
$\mathrm{O}(3)^{\prime}$ & $6 c$ & $3 \mathrm{~A}+3 \mathrm{E}_{1}+3 \mathrm{E}_{2}$ & 9 \\
$\mathrm{O}(4)^{\prime}$ & $6 c$ & $3 \mathrm{~A}+3 \mathrm{E}_{1}+3 \mathrm{E}_{2}$ & 9 \\
\hline & $6 c$ & Total (with split sites): & 90
\end{tabular}


Table S2. Raman activity in $P 6_{3} / \mathrm{m}$ space group.

\begin{tabular}{lccc}
\hline Atom & Site symmetry & Raman activity & Number of modes \\
\hline $\mathrm{Nd}(1)$ & $4 f$ & $\mathrm{~A}_{\mathrm{g}}+\mathrm{E}_{1 \mathrm{~g}}+\mathrm{E}_{2 \mathrm{~g}}$ & 3 \\
$\mathrm{Nd}(2)$ & $6 h$ & $2 \mathrm{~A}_{\mathrm{g}}+\mathrm{E}_{1 \mathrm{~g}}+2 \mathrm{E}_{2 \mathrm{~g}}$ & 5 \\
$\mathrm{Si}$ & $6 h$ & $2 \mathrm{~A}_{\mathrm{g}}+\mathrm{E}_{1 \mathrm{~g}}+2 \mathrm{E}_{2 \mathrm{~g}}$ & 5 \\
$\mathrm{O}(1)$ & $6 h$ & $2 \mathrm{~A}_{\mathrm{g}}+\mathrm{E}_{1 \mathrm{~g}}+2 \mathrm{E}_{2 \mathrm{~g}}$ & 5 \\
$\mathrm{O}(2)$ & $6 h$ & $2 \mathrm{~A}_{\mathrm{g}}+\mathrm{E}_{1 \mathrm{~g}}+2 \mathrm{E}_{2 \mathrm{~g}}$ & 5 \\
$\mathrm{O}(3)$ & $12 i$ & $3 \mathrm{~A}_{\mathrm{g}}+3 \mathrm{E}_{1 \mathrm{~g}}+3 \mathrm{E}_{2 \mathrm{~g}}$ & 9 \\
$\mathrm{O}(4)$ & $2 a$ & Total (without split sites): & 1 \\
\hline \multicolumn{5}{c}{$\mathrm{E}_{2 \mathrm{~g}}$} & Raman activity & Number of modes \\
\hline $\mathrm{Split}$ site & Site symmetry & $3 \mathrm{~A}_{\mathrm{g}}+3 \mathrm{E}_{1 \mathrm{~g}}+3 \mathrm{E}_{2 \mathrm{~g}}$ & 9 \\
\hline $\mathrm{O}(1)^{\prime}$ & $6 h$ & $3 \mathrm{~A}_{\mathrm{g}}+3 \mathrm{E}_{1 \mathrm{~g}}+3 \mathrm{E}_{2 \mathrm{~g}}$ & 9 \\
$\mathrm{O}(3)^{\prime}$ & $12 i$ & Total (with split sites): & 51
\end{tabular}

Table $\mathrm{S}_{3}$. Internal modes of $\mathrm{SiO}_{4}$ in $\mathrm{Pb}_{3}$ space group. The site group $\left(\mathrm{C}_{1}\right)$ is correlated to the factor group $\left(\mathrm{C}_{6}\right)$ as: $\mathrm{A}$ $\rightarrow A+B+E_{1}+E_{2}$.

\begin{tabular}{lccccc}
\hline Internal modes & Molecular group $\left(\mathrm{T}_{\mathrm{d}}\right)$ & Site group $\left(\mathrm{C}_{1}\right)$ & Factor group $\left(\mathrm{C}_{6}\right)$ & Raman activity & Number of modes \\
\hline$v_{1}$ & $\mathrm{~A}_{1}$ & $\mathrm{~A}$ & $\mathrm{~A}+\mathrm{B}+\mathrm{E}_{1}+\mathrm{E}_{2}$ & $\mathrm{~A}+\mathrm{E}_{1}+\mathrm{E}_{2}$ & 3 \\
$v_{2}$ & $\mathrm{E}$ & $2 \mathrm{~A}$ & $2 \mathrm{~A}+2 \mathrm{~B}+2 \mathrm{E}_{1}+2 \mathrm{E}_{2}$ & $2 \mathrm{~A}+2 \mathrm{E}_{1}+2 \mathrm{E}_{2}$ & 6 \\
$v_{3}$ & $\mathrm{~T}_{2}$ & $3 \mathrm{~A}$ & $3 \mathrm{~A}+3 \mathrm{~B}+3 \mathrm{E}_{1}+3 \mathrm{E}_{2}$ & $3 \mathrm{~A}+3 \mathrm{E}_{1}+3 \mathrm{E}_{2}$ & 9 \\
$v_{4}$ & $\mathrm{~T}_{2}$ & $3 \mathrm{~A}$ & $3 \mathrm{~A}+3 \mathrm{~B}+3 \mathrm{E}_{1}+3 \mathrm{E}_{2}$ & $3 \mathrm{~A}+3 \mathrm{E}_{1}+3 \mathrm{E}_{2}$ & 9 \\
\hline
\end{tabular}

Table $\mathrm{S}_{4}$. Internal modes of $\mathrm{SiO}_{4}$ in $\mathrm{Pb}_{3} / \mathrm{m}$ space group. The site group $\left(\mathrm{C}_{\mathrm{s}}\right)$ is correlated to the factor group $\left(\mathrm{C}_{6 \mathrm{~h}}\right)$ as: $A^{\prime} \rightarrow A_{g}+E_{2 g}+B_{u}+E_{1 u}$ and $A^{\prime \prime} \rightarrow B_{g}+E_{1 g}+A_{u}+E_{2 u}$.

\begin{tabular}{lccccc}
\hline $\begin{array}{l}\text { Internal } \\
\text { modes }\end{array}$ & $\begin{array}{c}\text { Molecular } \\
\text { group }\left(\mathrm{T}_{\mathrm{d}}\right)\end{array}$ & $\begin{array}{c}\text { Site group } \\
\left(\mathrm{C}_{\mathrm{s}}\right)\end{array}$ & Factor group $\left(\mathrm{C}_{6 \mathrm{~h}}\right)$ & Raman activity & Number of modes \\
\hline$v_{1}$ & $\mathrm{~A}_{1}$ & $\mathrm{~A}^{\prime}$ & $\mathrm{A}_{\mathrm{g}}+\mathrm{E}_{2 \mathrm{~g}}+\mathrm{B}_{\mathrm{u}}+\mathrm{E}_{1 \mathrm{u}}$ & $\mathrm{A}_{\mathrm{g}}+\mathrm{E}_{2 \mathrm{~g}}$ & 2 \\
$v_{2}$ & $\mathrm{E}$ & $\mathrm{A}^{\prime}+\mathrm{A}^{\prime \prime}$ & $\mathrm{A}_{\mathrm{g}}+\mathrm{E}_{2 \mathrm{~g}}+\mathrm{B}_{\mathrm{u}}+\mathrm{E}_{1 \mathrm{u}}+\mathrm{B}_{\mathrm{g}}+\mathrm{E}_{1 \mathrm{~g}}+\mathrm{A}_{\mathrm{u}}+\mathrm{E}_{2 \mathrm{u}}$ & $\mathrm{A}_{\mathrm{g}}+\mathrm{E}_{1 \mathrm{~g}}+\mathrm{E}_{2 \mathrm{~g}}$ & 3 \\
$v_{3}$ & $\mathrm{~T}_{2}$ & $2 \mathrm{~A}^{\prime}+\mathrm{A}^{\prime \prime}$ & $2 \mathrm{~A}_{\mathrm{g}}+2 \mathrm{E}_{2 \mathrm{~g}}+2 \mathrm{~B}_{\mathrm{u}}+2 \mathrm{E}_{1 \mathrm{u}}+\mathrm{B}_{\mathrm{g}}+\mathrm{E}_{1 \mathrm{~g}}+\mathrm{A}_{\mathrm{u}}+\mathrm{E}_{2 \mathrm{u}}$ & $2 \mathrm{~A}_{\mathrm{g}}+\mathrm{E}_{\mathrm{gg}}+2 \mathrm{E}_{2 \mathrm{~g}}$ & 5 \\
$v_{4}$ & $\mathrm{~T}_{2}$ & $2 \mathrm{~A}^{\prime}+\mathrm{A}^{\prime \prime}$ & $2 \mathrm{~A}_{\mathrm{g}}+2 \mathrm{E}_{2 \mathrm{~g}}+2 \mathrm{~B}_{\mathrm{u}}+2 \mathrm{E}_{\mathrm{uu}}+\mathrm{B}_{\mathrm{g}}+\mathrm{E}_{\mathrm{gg}}+\mathrm{A}_{\mathrm{u}}+\mathrm{E}_{2 \mathrm{u}}$ & $2 \mathrm{~A}_{\mathrm{g}}+\mathrm{E}_{\mathrm{gg}}+2 \mathrm{E}_{2 \mathrm{~g}}$ & 5 \\
\hline
\end{tabular}

Table $S_{5}$. Raman activity of possible oxygen interstitials in $P 6_{3} / m$ space group.

\begin{tabular}{lccc}
\hline $\mathrm{O}_{\text {int }}$ site symmetry & Coordinates & Raman activity & Number of modes \\
\hline $4 e$ & $($ o $о \mathrm{z})$ & $\mathrm{A}_{\mathrm{g}}+\mathrm{E}_{1 \mathrm{~g}}+\mathrm{E}_{2 \mathrm{~g}}$ & 3 \\
$2 b$ & $($ o o o $)$ & None & 0 \\
$6 h$ & $\left(x y^{1 / 4}\right)$ & $2 \mathrm{~A}_{\mathrm{g}}+\mathrm{E}_{1 \mathrm{~g}}+2 \mathrm{E}_{2 \mathrm{~g}}$ & 5 \\
\hline
\end{tabular}


Table S6. Internal modes of perturbed $\mathrm{SiO}_{4}$ in $\mathrm{Pb}_{3} / \mathrm{m}$ space group. The site group symmetry is lowered from $\mathrm{C}_{\mathrm{s}}$ to $C_{1}$, which is correlated to the factor group $\left(C_{6 h}\right)$ as: $A \rightarrow A_{g}+A_{u}+B_{g}+B_{u}+E_{1 g}+E_{1 u}+E_{2 g}+E_{2 u}$.

\begin{tabular}{lccccc}
\hline $\begin{array}{l}\text { Internal } \\
\text { modes }\end{array}$ & $\begin{array}{c}\text { Molecular } \\
\text { group }\left(\mathrm{T}_{\mathrm{d}}\right)\end{array}$ & $\begin{array}{c}\text { Site group } \\
\left(\mathrm{C}_{1}\right)\end{array}$ & Factor group $\left(\mathrm{C}_{6 \mathrm{~h}}\right)$ & Raman activity & Number of modes \\
\hline$v_{1}$ & $\mathrm{~A}_{1}$ & $\mathrm{~A}$ & $\mathrm{~A}_{\mathrm{g}}+\mathrm{A}_{\mathrm{u}}+\mathrm{B}_{\mathrm{g}}+\mathrm{B}_{\mathrm{u}}+\mathrm{E}_{1 \mathrm{~g}}+\mathrm{E}_{1 \mathrm{u}}+\mathrm{E}_{2 \mathrm{~g}}+\mathrm{E}_{2 \mathrm{u}}$ & $\mathrm{A}_{\mathrm{g}}+\mathrm{E}_{1 \mathrm{~g}}+\mathrm{E}_{2 \mathrm{~g}}$ & 3 \\
$v_{2}$ & $\mathrm{E}$ & $2 \mathrm{~A}$ & $\mathrm{~A}_{\mathrm{g}}+\mathrm{A}_{\mathrm{u}}+\mathrm{B}_{\mathrm{g}}+\mathrm{B}_{\mathrm{u}}+\mathrm{E}_{1 \mathrm{~g}}+\mathrm{E}_{1 \mathrm{u}}+\mathrm{E}_{2 \mathrm{~g}}+\mathrm{E}_{2 \mathrm{u}}$ & $2 \mathrm{~A}_{\mathrm{g}}+2 \mathrm{E}_{1 \mathrm{~g}}+2 \mathrm{E}_{2 \mathrm{~g}}$ & 9 \\
$v_{3}$ & $\mathrm{~T}_{2}$ & $3 \mathrm{~A}$ & $\mathrm{~A}_{\mathrm{g}}+\mathrm{A}_{\mathrm{u}}+\mathrm{B}_{\mathrm{g}}+\mathrm{B}_{\mathrm{u}}+\mathrm{E}_{1 \mathrm{~g}}+\mathrm{E}_{1 \mathrm{u}}+\mathrm{E}_{2 \mathrm{~g}}+\mathrm{E}_{2 \mathrm{u}}$ & $3 \mathrm{~A}_{\mathrm{g}}+3 \mathrm{E}_{1 \mathrm{~g}}+3 \mathrm{E}_{2 \mathrm{~g}}$ & 9 \\
$v_{4}$ & $\mathrm{~T}_{2}$ & $3 \mathrm{~A}$ & $\mathrm{~A}_{\mathrm{g}}+\mathrm{A}_{\mathrm{u}}+\mathrm{B}_{\mathrm{g}}+\mathrm{B}_{\mathrm{u}}+\mathrm{E}_{1 \mathrm{~g}}+\mathrm{E}_{1 \mathrm{u}}+\mathrm{E}_{2 \mathrm{~g}}+\mathrm{E}_{2 \mathrm{u}}$ & $3 \mathrm{~A}_{\mathrm{g}}+3 \mathrm{E}_{1 \mathrm{~g}}+3 \mathrm{E}_{2 \mathrm{~g}}$ & 9 \\
\hline & & & & Total: & 27
\end{tabular}

